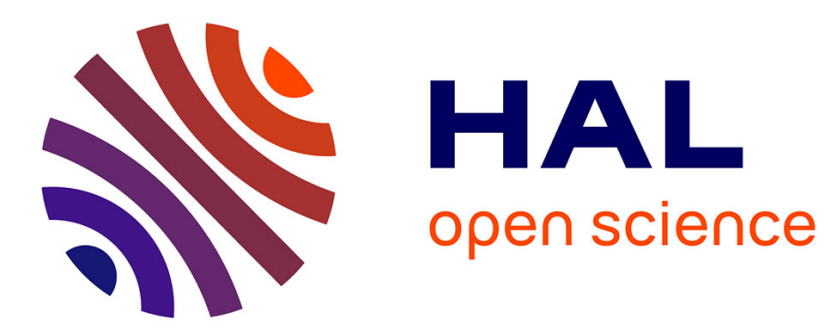

\title{
Le trueque argentin ou la question du fédéralisme monétaire (1995-2002) \\ Hadrien Saiag
}

\section{To cite this version:}

Hadrien Saiag. Le trueque argentin ou la question du fédéralisme monétaire (1995-2002). Revue Française de Socio-Economie, 2013, 12 (2), pp.69-89. 10.3917/rfse.012.0069 . halshs-02343413

\section{HAL Id: halshs-02343413 https://shs.hal.science/halshs-02343413}

Submitted on 11 Nov 2019

HAL is a multi-disciplinary open access archive for the deposit and dissemination of scientific research documents, whether they are published or not. The documents may come from teaching and research institutions in France or abroad, or from public or private research centers.
L'archive ouverte pluridisciplinaire HAL, est destinée au dépôt et à la diffusion de documents scientifiques de niveau recherche, publiés ou non, émanant des établissements d'enseignement et de recherche français ou étrangers, des laboratoires publics ou privés. 


\title{
LE TRUEQUE ARGENTIN OU LA QUESTION DU FÉDÉRALISME MONÉTAIRE (1995-2002)
}

\author{
Hadrien Saiag
}

\section{La Découverte I Revue Française de Socio-Économie}

\author{
2013/2 - n० 12 \\ pages 69 à 89
}

ISSN 1966-6608

Article disponible en ligne à l'adresse:

http://www.cairn.info/revue-francaise-de-socio-economie-2013-2-page-69.htm

Pour citer cet article :

Saiag Hadrien, « Le trueque argentin ou la question du fédéralisme monétaire (1995-2002) »,

Revue Française de Socio-Économie, 2013/2 n 12, p. 69-89. DOI : 10.3917/rfse.012.0069

Distribution électronique Cairn.info pour La Découverte.

(c) La Découverte. Tous droits réservés pour tous pays.

La reproduction ou représentation de cet article, notamment par photocopie, n'est autorisée que dans les limites des conditions générales d'utilisation du site ou, le cas échéant, des conditions générales de la licence souscrite par votre établissement. Toute autre reproduction ou représentation, en tout ou partie, sous quelque forme et de quelque manière que ce soit, est interdite sauf accord préalable et écrit de l'éditeur, en dehors des cas prévus par la législation en vigueur en France. II est précisé que son stockage dans une base de données est également interdit. 


\title{
Le trueque argentin ou la question du fédéralisme monétaire (1995-2002)
}

\author{
Hadrien SAIAG ${ }^{1}$ \\ CNRS/University of Pretoria (Human Economy Programme) \\ hadrien.saiag@gmail.com
}

\begin{abstract}
Cet article propose une réflexion sur le fédéralisme monétaire à partir de l'histoire des monnaies complémentaires en Argentine (trueque). Le premier réseau d'ampleur nationale (RGT, 1995-2000) incarne une forme possible de fédéralisme, caractérisée par la décentralisation de l'émission monétaire et le maintien de l'unicité de l'unité de compte. Ce modèle fut contesté par deux réseaux centralisant l'émission monétaire (RGT, 2001-2002 et RTZO, 2000-2002). La crise que connut l'ensemble de ces réseaux en 2002 souligne les implications éthiques et politiques de la question de l'émission monétaire.
\end{abstract}

\section{Considering monetary federalism from the Argentinian trueque (1995-2002)}

This paper presents a reflection on monetary federalism from the history of the Argentinian complementary currencies (trueque). The first national trueque system (RGT, 1995-2000) is analyzed as a form of monetary federalism. It is characterized by the decentralization of money-issuing and the uniqueness of the unit of account. This model has been challenged by two systems based on the centralization of money-issuing (RGT, 2001-2002 and RTZO, 2000-2002). The crisis that threatened the trueque in 2002 reveals ethical and political implications of the question of money-issuing.

Mots clés : fédéralisme, monnaie, Argentine, trueque, confiance

Keywords: federalism, money, Argentina, trueque, trust

\section{Introduction}

L'histoire monétaire de l'Argentine des années 1990 au début des années 2000 déstabilisera ceux qui voient dans la monnaie un artefact unifié visant à faciliter les échanges. L'apparente complexité

${ }^{1}$ Cet article a été rédigé lorsque j'étais chercheur post-doctoral au sein du programme Human Economy, University of Pretoria. Des versions antérieures de ce texte ont bénéficié des commentaires des participants au séminaire des doctorants de I'IRISSO ainsi que de la lecture attentive de Jérôme Blanc, Keith Hart, André Orléan, Jean-Michel Servet, Bruno Théret et Florence Weber. Qu'ils en soient remerciés. Je reste cependant le seul responsable des éventuelles erreurs que comporte ce texte. 
de la situation est due à la pluralité des unités de compte et des émetteurs de moyens de paiement : ainsi, les transactions pouvaient être évaluées et réglées en peso (monnaie nationale), en dollar états-unien, en monnaie fiscale nationale (Lecop), en monnaies provinciales (dans 16 des 23 provinces argentines [Théret, 2012]), ou encore en monnaies dites sociales (trueque). Cette pluralité est souvent assimilée à un symptôme de la crise économique aiguë qui toucha le pays en 2001 et 2002, les monnaies fiscales et le trueque étant associés à des monnaies de nécessité visant à pallier la pénurie monétaire (peso et dollar) induite par la fuite des capitaux dont fut victime l'Argentine (1998-2001). La fin de l'expérience des monnaies fiscales en 2003 et la crise que connut le trueque en 2002 marqueraient alors un retour à la normalité : I'unicité monétaire à l'échelle du territoire national.

Cette lecture est cependant réductrice, car elle ne tire aucun enseignement de la récurrence historique de la pluralité monétaire en Argentine [Théret, 2012]. Certes, la pluralité monétaire des années 1990 et 2000 s'inscrit dans le cadre de la crise du régime dit de « convertibilité » (1991-2002), qui faisait du peso un équivalent du dollar états-unien pour les transactions internes : les contraintes propres à ce régime monétaire se sont traduites par un accroissement des inégalités de revenus dès 1995 [Paraje, 2005], puis par une pénurie de monnaie nationale. Une analyse détaillée de cette expérience révèle cependant un enseignement d'une portée plus générale : la double stratification de la société argentine en sphères transactionnelles. La dislocation des taux de change entre les monnaies ${ }^{2}$ souligne d'abord qu'il existe une pluralité de types de dettes (marchandes, fiscales, domestiques, etc.) dont les circuits ne sont pas nécessairement unifiés [Blanc, 2000 ; Servet, Théret et Yildirim, 2008 ;Zelizer, 2005 (1994) ; Parry et Bloch (dir.), 1989]. Un examen attentif des monnaies fiscales provinciales [Théret et Zanabria, 2009] et du trueque révèle en outre une forte fragmentation territoriale des sphères transactionnelles.

Cet article propose une réflexion relative à la fragmentation territoriale des sphères transactionnelles à partir de l'histoire des principaux réseaux de trueque d'ampleur nationale, de 1995 à $2002^{3}$. Ce terme désigne les multiples expériences d'émission de monnaie papier de très petite dénomination (utilisées par des populations à bas revenus), libellées dans une unité de compte propre (crédito), qui drainent une part considérable de l'économie urbaine de l'occasion à travers des places de marché hebdomadaires (ferias). Rapidement, ces expériences se sont articulées autour d'un réseau d'ampleur nationale, le Réseau Global de Trueque (RGT), avant que celui-ci implose, donnant naissance au Réseau de Trueque de la Zone Ouest [de Buenos Aires] (RTZO) et au Réseau de Trueque Solidaire (RTS). Ces trois réseaux entrèrent en crise en 2002.

L'analyse proposée ici met l'accent sur la dimension institutionnelle du trueque, pour deux raisons. D'abord, afin de soulever la question du fédéralisme monétaire,

\footnotetext{
${ }^{2}$ En règle générale, les moyens de paiement libellés dans les différentes monnaies n'étaient pas convertibles à la parité.

${ }^{3}$ Une telle restriction de l'objet d'étude appelle deux précisions : d'une part, le trueque n'a pas disparu après sa crise en 2002 [Plasencia et Orzi, 2007; Gómez, 2008 ; Saiag, 2011] ; d'autre part, les réseaux nationaux ont cohabité avec des réseaux provinciaux. Cependant, les informations disponibles se concentrent sur les réseaux nationaux de 1995 à 2002.
} 
largement ignorée ${ }^{4}$. Ainsi, l'histoire du trueque peut être lue comme celle des tensions entre deux types d'architectures institutionnelles opposées : le RGT (jusqu'en 2001) puis le RTS incarnent un modèle de « fédéralisme monétaire », qui reconnaît et articule les différentes sphères transactionnelles les unes aux autres à travers la décentralisation de l'émission monétaire et le maintien de l'unicité de l'unité de compte ; le RGT (à partir de 2001) et le RTZO incarnent quant à eux deux modalités contrastées de centralisation de l'émission monétaire, qui nient la fragmentation territoriale des sphères transactionnelles. Ensuite, car l'analyse institutionnelle permet de souligner les dimensions politiques de la monnaie, à travers les formes de confiance mises à mal lors de la crise du trueque (2002).

L'argument est présenté en quatre temps. La première section précise la méthode employée. Les sections suivantes montrent que la tension entre fédéralisme et centralisation monétaire traverse l'histoire du trueque. Elles décrivent l'émergence d'une forme de fédéralisme monétaire à travers la constitution du RGT (section 2), puis sa contestation par des formes de centralisation de l'émission monétaire (section 3). La quatrième section analyse les crises que connurent les réseaux d'ampleur nationale comme des crises de confiance éthique, dont les fondements sont intimement liés aux modalités d'émission monétaire propre à chaque réseau.

\section{Un raisonnement par hypothèses}

La reconstitution de l'histoire du trueque exige de prendre quelques précautions méthodologiques. Son histoire " officielle » a été écrite par ses fondateurs à travers la publication de brochures, d'articles dans la presse et d'actes de colloques universitaires [Cortesi, 2003 ; Laporte, 2003 ; Perez-Lora, 2003 ; Primavera, 2003 ; Primavera, Covas et de Sanzo, 1998 ; Ravera, 2003 ; Sampayo, 2003]. Or ce type de récit pose problème car les informations que l'on y trouve sont parfois contradictoires et souvent trop vagues pour permettre de tirer, à elles seules, des conclusions solides.

Afin de dépasser cet obstacle, nous proposons d'étayer la reconstitution de l'histoire du trueque à travers un raisonnement par hypothèses [Servet, 2009 : chapitre 4]. Puisqu'il n'est possible d'observer que des fragments de l'histoire du trueque, il convient de proposer un nombre réduit d'hypothèses qui, si elles ne sont pas contredites par les fragments dont nous disposons, doivent être acceptées faute de mieux, comme une « approximation hypothétique » de la réalité [op. cit.]. Dans cette optique, le récit des fondateurs du trueque servira de point de départ : afin d'avancer des hypothèses rigoureuses, il sera confronté à un éventail de sources les plus diverses possibles. Les éléments vers lesquels l'ensemble des sources convergent ainsi que ceux qui ne peuvent être réfutés à partir d'éléments probants seront, faute de mieux, acceptés comme des approximations de la réalité.

Ainsi, cet article croise les témoignages des fondateurs du trueque avec des sources d'origines multiples. En l'absence de dépôt d'archives, nous mobilisons essentiellement un document écrit qui atteste directement de l'histoire du trueque : le bulletin

${ }^{4}$ Voir les exceptions notables d'Arnsperger [2011], Olivera [1992] et Théret et Zanabria [2007]. 
fédéral de créditos (Boletín federal de créditos), $n^{0} 5^{5}$, publié en septembre 2001 par l'organisme chargé de contrôler les modalités d'émission monétaire du RTS. Ce document offre une description détaillée des étapes ayant mené à l'élaboration d'un mécanisme d'émission monétaire propre à ce réseau et atteste des conflits opposant les différents réseaux d'ampleur nationale.

Un nombre important d'informations relatives à l'histoire du trueque est également issu d'une lecture fine de la littérature (articles, ouvrages, thèses et mémoires). Lorsqu'elles sont issues d'enquêtes de terrain, ces publications exposent des données « brutes » concernant I'histoire du trueque, à travers des extraits d'entretiens ou de brèves descriptions. Nous disposons également de publications de qualité concernant l'évolution des types de participants au trueque [Gonzales-Bombal, 2002 ; Salles, 2006 ; Leoni, 2003]. Enfin, la publication des actes d'un colloque ayant porté sur le trueque [Hintze (dir.), 2003] propose une revue détaillée des principaux quotidiens nationaux (et de quelques quotidiens provinciaux) et offre des éléments permettant d'analyser la nature de la crise que connut le trueque fin 2002.

À ces sources écrites s'ajoutent deux témoignages oraux, recueillis lors d'une enquête de terrain menée entre août et décembre 2009 : le témoignage de la fondatrice du trueque dans le sud de la province de Santa Fe, Elena, et le témoignage de la responsable du trueque dans les banlieues nord de Rosario (principale agglomération de ladite province), Marita. Ces entretiens permettent de mieux comprendre le fonctionnement institutionnel du RGT puis du RTS, car Marita et Elena ont participé à l'ensemble des réunions de l'organe chargé du contrôle de l'émission monétaire de ces réseaux (la « commission interzonale »), de 1999 à 2002.

\section{De l'unification à la fragmentation de l'émission monétaire : l'émergence d'une forme de fédéralisme monétaire}

Le premier réseau de trueque d'ampleur nationale (le RGT) prend forme par étapes, entre 1995 et 2000. Durant cinq années, le trueque connaît une croissance continue mais contenue du nombre de ses participants, corrélatif de la paupérisation de la classe moyenne argentine [Kessler, 1999], engendrée notamment par la flexibilisation du marché du travail. Cette période est également marquée par une série de transformations de la structure institutionnelle du trueque à travers des processus successifs de centralisation puis de fragmentation de l'émission monétaire, qui ont donné naissance à une forme de fédéralisme monétaire.

Le récit des fondateurs du trueque portant sur la période 1995-2000 est relativement peu contesté [Primavera, Covas et de Sanzo, 1998 ; Cortesi, 2003 ; Primavera, 2003]. Ainsi, il est admis par tous que l'événement marquant sa création fut une réunion de voisins en vue d'échanger les excédents de leurs productions domestiques dans le garage de Carlos de Sanzo, à Bernal (sud de Buenos Aires). Ce dernier est considéré comme l'un des fondateurs du trueque, avec ses associés Rubén Ravera et Horacio 
Covas, principaux responsables d'une organisation de protection de l'environnement : le Programme d'autosuffisance régional. À cette date, il n'existait qu'une seule place de marché hebdomadaire (feria). Celle-ci était précédée par une discussion qui, selon les fondateurs, visait à « réinventer le marché ». Les transactions n'avaient lieu que dans un second temps. Les participants entraient tour à tour afin de déposer les objets qu'ils souhaitaient proposer. Leur valeur, estimée à l'aide d'une unité de compte propre, le crédito, était annotée sur des « cartes d'échange » (tarjetas de intercambio) personnelles. Ensuite seulement, ils choisissaient les biens qu'ils souhaitaient acquérir. Leur valeur était de nouveau inscrite sur la même " carte d'échange ». À la fin de chacune des ferias, les soldes de chacun des participants étaient centralisés sur un cahier de compte, puis informatisés.

Des systèmes relativement proches semblent avoir été mis en place jusqu'en septembre 1996 [Echavarri, 2003]. Tantôt les dettes étaient compensées bilatéralement, tantôt multilatéralement; mais celles contractées dans différentes ferias ne pouvaient être compensées. Le trueque prenait alors la forme d'un ensemble de systèmes de " compensation pure » [Blanc, 2000, p. 25] indépendants les uns des autres, proches du système de " compensation dynamique multilatérale des dettes et des créances » que connaissent la plupart des SEL en France [Servet, 1999, p. 183].

Entre septembre et décembre 1996, deux éléments clés transformèrent le système en place et donnèrent naissance au RGT [Hintze et al., 2003 ; Primavera, 2003]. Avec l'accroissement du nombre de participants, la gestion des systèmes de compensation pure devenait de plus en plus lourde. Ceux-ci furent donc remplacés par l'émission de billets libellés en créditos. Dans un premier temps, les moyens de paiement étaient émis localement et n'étaient valables que dans leur feria d'origine. Cependant, cet événement fut rapidement suivi de l'acte inaugural du RGT : la centralisation de l'émission monétaire, à travers le remplacement des moyens de paiement par ceux émis par les fondateurs du trueque, réunis dans le « Conseil consultant » du PAR. Dès lors, il était possible de passer d'une place de marché à l'autre sans changer de monnaie, au prix d'une double unification : celle du système de compte (les dettes étaient comptabilisées en crédito sur l'ensemble des ferias) et celle de l'émission des moyens de paiement (chaque nouveau participant recevant 50 créditos). Les places de marché sont désormais nommées « nodos ${ }^{6} »$.

Cependant, le temps de la centralisation de l'émission monétaire fut bref. On observe, avant mai 1997, un mouvement de fragmentation de l'émission monétaire qui transforma le RGT en un « réseau de réseaux ». Les nodos appartenant au RGT, lesquels étaient alors concentrés dans Buenos Aires et sa banlieue, furent regroupés en différentes zones selon leur situation géographique : les zones Sud, Capitale, Ouest et Nord. Par la suite le trueque s'étendra à d'autres provinces, mais cela n'altéra pas fondamentalement le fonctionnement du RGT : de nouvelles zones furent créées (Córdoba, Rosario et Sud de la province de Santa Fe, Entre Ríos, Catamarca, etc. [Luzzi, 2005, p. 49]). La création et la distribution des moyens de paiement étaient désormais effectuées au niveau de chacune des zones. Bien qu'émis de manière

${ }^{6}$ Ce terme se réfère aux mailles d'un filet, produisant l'image d'une organisation en réseau (red). 
décentralisée et étant clairement reconnaissables (les dessins ainsi que les couleurs des billets variaient selon les zones), tous les moyens de paiement étaient valables dans l'ensemble du réseau. L'élément clé de ce système était la Commission interzonale (Comisión Interzonal) qui, composée de représentants des différentes zones du RGT, était chargée du contrôle du respect des normes d'émission monétaire.

Ces normes ont émergé progressivement afin de répondre à l'enjeu principal posé par la décentralisation de l'émission monétaire : préserver l'unicité du compte dans l'ensemble du réseau. II semble en effet que ce dernier fut dans un premier temps en proie à de forts différentiels d'inflation d'une zone à l'autre, dus à l'absence de règle quantitative encadrant l'émission monétaire décentralisée. Ainsi, le Boletín federal de créditos daté de septembre 2001 précise que la quantité de créditos en circulation

« ne peut être arbitraire ou très différente d'une zone à l'autre à cause des distorsions que cela peut engendrer.

Si la quantité de créditos est trop faible [...], les échanges sont entravés et si cette quantité est excessive il se produit un phénomène bien connu, l'inflation (les prix montent). Mais si, en outre, il y a beaucoup de créditos en circulation dans une zone et peu, ou la quantité juste suffisante dans une autre, il se produit un phénomène de désapprovisionnement de cette dernière, puisque les participants mobiles vont tenter de vendre leurs produits dans la zone en proie à l'inflation afin d'obtenir de meilleurs revenus » [Boletín federal de créditos, septembre 2001, p. 4].

Or de trop fortes disparités dans la fixation des prix entre les zones font courir au réseau un risque d'implosion par fragmentation du système de compte : d'une part, de forts différentiels d'inflation peuvent se traduire, in fine, par l'apparition de taux de change non paritaires entre créditos émis par différentes zones ; d'autre part, les problèmes engendrés par ces différentiels peuvent conduire certaines zones à se retirer du réseau. Le Boletín federal de créditos ne faisant pas état de ce type de situation, il semble que le RGT a réussi à éviter une telle implosion jusqu'à l'an 2000. Cependant, les entretiens retranscrits par Echavarria [2003] soulignent qu'il s'agit d'un risque inhérent à l'architecture institutionnelle adoptée le RGT. Ils portent sur l'histoire d'un réseau de la province de Córdoba (le Red Nacional de Trueque - RNT), mais il est raisonnable d'émettre I'hypothèse que le RGT était en proie à des risques analogues, étant donné l'isomorphisme institutionnel existant entre les deux réseaux (le RNT ayant calqué l'organisation du RGT à l'échelle d'une province). L'auteure précise que la disparité des critères d'émission monétaire entre les quatre zones constituant le RNT (Córdoba, Santa Maria, Sierras Chicas et Punilla) s'est traduite par de forts différentiels d'inflation entre zones, qui ont permis aux participants les plus mobiles de mettre en œuvre des pratiques d'arbitrage aboutissant au désapprovisionnement des zones à faible inflation. Ce processus a probablement pris une ampleur considérable, puisque les zones Punilla et Santa Maria décidèrent de se séparer du RNT en refusant dorénavant les créditos des zones Córdoba et Sierras Chicas.

Afin d'éviter l'implosion, il était donc nécessaire au RGT d'adopter un mécanisme permettant de limiter les différentiels d'inflation entre zones. Face à ces problèmes, la Commission interzonale ne propose pas de centraliser l'émission monétaire (qui, in 
fine, aurait unifié les critères d'émission et de distribution). En effet, elle se montre attachée à la décentralisation de l'émission monétaire, sur la base de deux arguments. Le premier réside dans le refus de la soumission à un pouvoir central et la promotion du développement local : « [...] la décentralisation de l'édition des créditos a pour objet de fortifier le développement local et d'éviter la dépendance vis-à-vis d'un pouvoir centralisé qui édite et gère [maneje] le crédito. » [Commission fédérale de créditos, septembre 2001, p. 1] Le second argument insiste sur l'hétérogénéité des besoins monétaires sur le territoire argentin :

« II n'est pas possible d'établir des critères [d'émission monétaire] rigides [...], étant donné qu'il existe diverses réalités dans le pays. [...] l'important est que la zone [...] utilise sa faculté de pouvoir émettre ses propres créditos avec un système de contrôle qui garantisse la transparence. Cette autonomie [...] permettra à la zone ou à la région de planifier et de dynamiser son économie. » [Ibid., p. 2]

Dès lors, on assiste à un renforcement du fédéralisme monétaire, à travers l'établissement d'un cadre octroyant à chaque zone quelque latitude tout en les soumettant toutes à une même contrainte:

"La décentralisation de l'édition est synonyme d'autonomie, de liberté et de souveraineté [...]. Mais la décentralisation [...] nécessite la mise en place de mécanismes qui permettent l'utilisation de tous les créditos dans l'ensemble du réseau, ce qui signifie rechercher des mécanismes d'unification des usages de la diversité des créditos. » [lbid., p. 2 ; souligné par moi-même.]

Ainsi, les zones se sont engagées « à établir une limite de 100 créditos par membre » [ibid., p. 3], fixant ainsi une limite quantitative à l'émission décentralisée des créditos. Dès lors, l'importance de la Commission interzonale dans l'architecture institutionnelle du RGT se trouve accrue : elle doit désormais veiller au respect de cette nouvelle norme quantitative, ainsi que du découpage territorial opéré par le réseau (aucune zone ne devant émettre sur le territoire d'une autre zone). Il s'agit donc bien d'une forme possible de fédéralisme monétaire, dans laquelle les entités fédérées (les zones) s'auto-soumettent à un ensemble de règles visant à assurer l'unité (du compte) dans la diversité (des émetteurs de moyens de paiement).

\section{Fédéralisme contre centralisation de l'émission monétaire}

À partir de l'année 2000, le RGT fut l'objet de deux importantes scissions, qui donnèrent naissance au RTZO puis au RTS. Ces événements remirent en cause le modèle de fédéralisme monétaire adopté par le RGT, en lui opposant deux formes contrastées de centralisation de l'émission monétaire. Cependant, le fédéralisme monétaire fut maintenu coûte que coûte au sein du RTS.

\subsection{Une double contestation du fédéralisme monétaire}

Les origines des tensions internes au RGT ayant conduit à son implosion sont intimement liées à la contestation du principe de décentralisation de l'émission monétaire. Jusqu'en 2001, les fondateurs du trueque contrôlaient la zone sud de la Région 
métropolitaine (Buenos Aires et sa banlieue). Ils étaient, à ce titre, responsables de l'émission monétaire de la zone, mais furent rapidement accusés de ne pas respecter les règles établies par la Commission interzonale. Ils ont, par exemple, enfreint le principe de décentralisation de l'émission monétaire depuis 1997 en se chargeant de l'émission des moyens de paiement utilisés dans la zone de Mendoza de 1997 à 1999 [McClanahan, 2003, p. 90-96]. Les tensions avec les représentants des autres zones se sont accrues après leur décision de mettre à disposition de toute personne désirant ouvrir un nouveau nodo des créditos émis par leurs soins, afin d'accélérer l'expansion territoriale du réseau. Les détracteurs de cette politique (connue sous le nom de franchise sociale) accusèrent les fondateurs du trueque de chercher à s'enrichir en faisant payer un seigneuriage trop important : 50 créditos étaient « vendus » aux adhérents 3 pesos alors que les coûts d'impression étaient estimés à une vingtaine de centimes [Luzzi, 2005, p. 50].

Cependant, ceux qui se sont séparés du RGT s'accordent à situer le cœur du conflit au sein de la Commission interzonale [Sampayo, 2003 ; Cortesi, 2003 ; Perez-Lora, 2003 ; Primavera, 2003]. Les tensions évoquées précédemment culminèrent en septembre 2000 , lorsque les fondateurs quittèrent la réunion de ladite commission sans répondre aux demandes d'éclaircissement de leurs comptes [Luzzi, 2005, p. 50]. Les représentants de chaque zone étaient censés présenter un bilan (balance) présentant le détail des nouvelles émissions monétaires (numéro des billets et leur valeur faciale), la liste des nodos et le nombre d'adhérents de la zone. Or le bilan présenté par les fondateurs du trueque ne comportait que la quantité de créditos émise, sans en mentionner l'allocation. En outre, les autres zones les accusaient d'avoir mis en place un système de double numérotation et d'émettre au-delà des quantités annoncées. À partir de ce moment, il semble que les représentants de la zone sud de Buenos Aires n'aient plus pris part aux réunions de la Commission interzonale. Quelques mois plus tard, la zone ouest cessa de participer à la Commission interzonale et prit son autonomie vis-à-vis du RGT en créant le RTZO [Sampayo, 2003]. Les autres zones formèrent formellement le Réseau de Trueque Solidaire (RTS) en avril 2001 et les fondateurs du trueque réorganisèrent fondamentalement le RGT. Le RGT et le RTZO optèrent pour deux modes de centralisation de l'émission monétaire, qui remirent en question le fédéralisme monétaire porté par le RGT jusqu'en 2000.

Le RTZO opta pour une forme de centralisation de l'émission monétaire au service exclusif du développement de la banlieue ouest de Buenos Aires. Selon Fernando Sampayo, qui anima ce réseau jusqu'à sa mort en 2009, l'objet premier du RTZO était d'offrir un palliatif à la pauvreté à travers une gestion optimisée et centralisée du trueque à grande échelle [Gómez, 2008, p. 131-132]. Afin de remplir cet objectif, le réseau a d'abord ouvert ses portes à de nombreux petits commerçants. II a ensuite développé une capacité productive propre à travers la constitution de quatre ateliers produisant des aliments industriels destinés à être proposés lors des ferias (pâtes et pâte à pizza) et les meubles nécessaires au bon déroulement de ces dernières [Sampayo, $2003^{7}$ ]. Afin de remplir l'objectif qu'il s'était fixé, le RTZO opta pour la 
centralisation de l'émission monétaire, car elle permettait d'assurer son approvisionnement en matières premières, à travers l'ouverture de nodos dans plusieurs provinces argentines :

« Désormais, tout comme le PAR [RGT], les nodos du RTZO se trouvent principalement à l'ouest de Buenos Aires [...], mais il en possède certains à l'intérieur de la province de Buenos Aires [banlieue de Buenos Aires non comprise], sur la côte atlantique, dans les provinces de Mendoza, Córdoba, Corrientes, Tucuman et Misiones. Cela est dû à la nécessité d'entretenir un contact direct avec les producteurs des matières premières ou des produits régionaux qui sont indispensables à la production d'aliments. » [Sampayo, 2003, p. 199]

Imposer aux nodos provinciaux du RTZO la monnaie de ce réseau, principalement utilisée dans les banlieues ouest de Buenos Aires, les obligeait à orienter leurs transactions vers ceux-ci et, par conséquent, à leur fournir les matières premières dont ils avaient besoin. II s'agissait donc de contraindre les producteurs de certaines zones rurales jugées stratégiques à échanger avec une zone urbaine dans laquelle les populations à bas revenus faisaient face à une pénurie alimentaire.

Le fonctionnement du RGT fut quant à lui transformé en profondeur à partir de la scission du RTS en 2001. L'émission monétaire y était entièrement centralisée : le " conseil accesseur » du PAR, formé par les fondateurs du trueque, était le seul organe de décision en la matière et ne devait rendre de compte à personne. Plus fondamentalement, la stratégie adoptée par le RGT rompt avec la division territoriale prévalant avant les différentes scissions : à partir de 2000, il s'étend bien au-delà de la zone Sud de Buenos Aires et tente de concurrencer le RTS en ouvrant des nodos sur l'ensemble du territoire $\operatorname{argentin}^{8}$. La retranscription par Fabiana Leoni d'un entretien avec la coordinatrice d'un nodo situé au nord de Buenos Aires et ayant intégré le RGT jusqu'en 2002 permet d'entrevoir l'organisation monétaire du réseau :

"Nous franchisions ${ }^{9}$ à Rodriguez [banlieue ouest de Buenos Aires]. C'était un chaos et je me disais : "ce n'est pas possible qu'ils nous traitent si mal alors que nous les maintenons [siendo que nosotros los estamos manteniendo]." Nous allions retirer un numéro à $4 \mathrm{~h}$ du matin et ceux qui venaient de La Bernaleza avec les caisses de créditos à franchiser commençaient à s'occuper de nous à $9 \mathrm{~h}$. Nous avons dû attendre jusqu'à cinq ou six heures de l'après-midi avant de sortir, et en sortant... ils nous ont maltraités [...]. Tout cela m'a tellement indignée... » (Maria Inés, septembre 2003, José C. Paz, retranscrit dans Leoni [2003, p. 25-26], souligné par moi-même.)

Ainsi, les responsables du RGT avaient installé des relais au-delà de la zone originelle d'influence des fondateurs (le sud de Buenos Aires). L'impression des billets libellés en créditos devait avoir lieu à Bernal, siège du PAR, étant donné que ceux qui les y

\footnotetext{
${ }^{8}$ De telles ouvertures de nodos sont documentées concernant les régions Mar y Sierras [Pérez-Lora, 2003], Córdoba [Echavarri, 2003], Mendoza [Hintze et al., 2003], Santa Fe (entretiens avec Elena et Marita), la zone Nord de Buenos Aires [Leoni, 2003], la province de Catamarca [Verón Ponce et al., 2003] et celle de Misiones [Saiag, 2011, p. 52-63].

${ }^{9}$ Tel est le terme utilisé afin de qualifier l'octroi de créditos aux coordinateurs du RGT contre des pesos.
} 
apportaient « venaient de La Bernaleza », ancienne usine désaffectée et réhabilitée en siège du RGT à partir de 2001. Ainsi, il semble que le groupe fondateur contrôlait directement (et presque physiquement) le processus d'émission des créditos. Cet entretien confirme également que les créditos étaient octroyés contre des pesos, puisque Maria Inés estime qu'elle "maintenait » la structure par ses demandes de créditos. L'accent semble donc avoir été mis sur l'extension territoriale du réseau afin de maximiser les revenus tirés du monnayage.

\subsection{Le RTS, héritier du fédéralisme monétaire}

L'élément fondamental dans la structuration du trueque autour de trois réseaux à échelle nationale à partir de 2001 est, selon nous, la remise en question par le RGT et le RTZO du fédéralisme monétaire promu par le RTS et son maintien coûte que coûte dans ce dernier. En effet, tous les éléments dont nous disposons confirment que le RTS a bel et bien maintenu le principe de décentralisation de l'émission monétaire, malgré les multiples attaques dont il a été la cible.

Leélément clé de l'organisation spatiale du RGT puis du RTS était la Commission interzonale, chargée de garantir la décentralisation de l'émission monétaire et l'unicité du compte, à travers la production d'un document nommé balance («bilan »). Avant chaque réunion de la Commission interzonale, chaque zone devait établir son propre balance, en précisant la couleur des billets, le nom de chaque nodo, la quantité de créditos qui leur avait été accordée et, enfin le nombre de membres de chacun. Le « bilan » du réseau était alors élaboré à partir de la juxtaposition des balances des différentes zones. Ce document était au cœur des discussions lors des réunions de la Commission interzonale car il incarnait les principes permettant l'émission décentralisée des moyens de paiement au sein du réseau. Trois points méritent d'être soulignés à ce titre. Premièrement, le balance permettait de vérifier que le ratio maximal d'émission de créditos par participant (établi par la Commission interzonale) était bien respecté par chaque zone, puisqu'il faisait état de la numérotation des billets nouvellement émis et précisait leurs valeurs nominales. Deuxièmement, il permettait de contrôler le respect des normes de fragmentation territoriale de l'émission monétaire propre au RGT puis au RTS, car chaque zone devait préciser les nodos auxquels les moyens de paiement nouvellement émis étaient remis. Ce faisant, le balance permettait de vérifier que chaque zone émettait dans le territoire qui lui était imparti et qu'un seul crédito était émis par zone. Enfin, ce document énumérait les créditos à accepter dans l'ensemble du réseau. II constituait pour beaucoup la matérialisation de l'existence du réseau, car il permettait aux coordinateurs n'ayant jamais participé aux réunions de la Commission interzonale de savoir de quelles zones il était constitué et quels billets devaient être acceptés localement.

\section{3. Émission monétaire ou transformation sociale?}

La lecture des divergences entre les principaux réseaux de trueque qui vient d'être proposée ne fait pas l'unanimité. D'autres lectures mettent l'accent sur la dimension démocratique du fonctionnement du RTS et de ses ambitions en termes de transformation sociale, alors que ces deux dimensions auraient été moins marquées, voire totalement absentes au sein du RGT et du RTZO [Hintze (dir.), 2003 ; Primavera, 
2003 ; Montillet, 2006 ; Powell, 2006]. Ces lectures ne sont pas nécessairement contradictoires avec la nôtre, pour autant que ces caractéristiques se retrouvent dans les modalités d'émission des moyens de paiement : la question de l'émission des moyens de paiement est éminemment politique. Néanmoins il convient de prendre ses distances par rapport à une lecture de l'histoire du trueque qui consisterait à dissocier les dimensions monétaires et démocratiques des réseaux. Les enjeux intellectuels et politiques sont grands : dissocier ces aspects reviendrait à sous-entendre que la monnaie est neutre du point de vue politique (en termes d'enjeux démocratiques et distributifs). Or l'originalité du RTS est précisément d'avoir intégré la dimension démocratique aux modalités d'émission des moyens de paiement, à travers les étapes successives menant vers la constitution du balance du réseau. En effet, nous n'avons pas connaissance d'argument probant qui permettrait de dissocier clairement les dimensions militante et monétaire du trueque [Saiag, 2011, p. 157-163].

La dimension politique des modalités d'émission monétaire se donne également à voir dans les ressorts de la confiance propres au RTS. Cette dernière ne se limite pas à la dimension routinière des transactions (la « confiance méthodique » dans la terminologie d'Aglietta et al. [1998]) : elle révèle également la dimension holiste de la monnaie à travers ses dimensions hiérarchiques et éthiques [ibid.]. Ainsi, la Commission interzonale incarne la confiance hiérarchique en tant qu'institution qui, sans participer directement aux transactions, est chargée de faire respecter les règles monétaires, à travers le contrôle de l'émission des moyens de paiement et de la procédure à suivre afin d'accepter une nouvelle zone dans le réseau. La confiance éthique, quant à elle, est plus difficile à cerner. Elle renvoie à l'adéquation des institutions qui étayent les usages du crédito (modalités d'émission monétaire, dimensions symboliques, etc.) avec le projet politique qui fonde le RTS : pour que le crédito jouisse d'une confiance éthique, il doit traduire monétairement un projet politique plus large, dans lequel se retrouvent ses utilisateurs [Théret, 2008, p. 818]. Ainsi appréhendée, la confiance éthique reste le plus souvent invisible, tacite, en dehors des périodes de crise monétaire [Théret, 2007]. Le conflit qui donna naissance au RTS permet néanmoins d'en cerner les contours. L'expulsion du groupe fondateur est due à leur non-respect d'une règle monétaire de première importance : la présentation du balance de chaque zone auprès de la Commission interzonale. Cet événement a poussé la Commission interzonale à préciser les fondements de la règle qui avait été transgressée : selon celle-ci, ne pas présenter de balance était incompatible avec la participation de la zone en question au sein du RGT (puis du RTS), car cela remettait en cause l'autonomie régionale et faisait courir le risque d'une soumission des zones à un pouvoir central et autoritaire. Ce faisant, l'architecture institutionnelle du RTS est bien la traduction d'un projet politique qui prend corps à travers la décentralisation de l'émission monétaire ; projet politique auquel s'opposent le RGT et le RTZO.

\section{Une crise révélatrice des fondements éthiques de la confiance}

Les tensions entre modèles fédératifs et centralisés ont également marqué la crise que connut l'ensemble des réseaux de trueque. L'approfondissement de la crise argentine, marquée par l'accentuation de la pénurie de moyens de paiement libellés 
en peso en 2001 (corralito ${ }^{10}$ ) puis la sortie du régime de convertibilité (janvier 2002), s'est d'abord traduit par un afflux massif de participants au sein du trueque: on estime qu'en mai 2002 environ 2500000 personnes participaient directement au trueque (tous réseaux confondus), contre 600000 l'année précédente [Ovalles, $2002^{11}$ ]. Le trueque fut alors investi par une nouvelle population : les dits " pauvres structuraux », pour lesquels la situation d'exclusion est chronique et non liée aux dégradations du marché de l'emploi des années 1990 [Kessler, 1999 ; Gonzales-Bombal, 2002 ; Salles, 2006]. Cependant, cet afflux massif de participants a été rapidement suivi d'une chute brutale que reconnaissent les responsables des divers réseaux de trueque [Ravera, 2003 ; Sampayo, 2003 ; Cortesi, 2003]. Ainsi, on estime qu'en 2003 le nombre de participants avait baissé de $50 \%$ [Luzzi, 2005, p. 55-56] à $90 \%$ [Hintze et al., 2003, p. 69] par rapport à son maximum, atteint durant l'année 2002.

Selon nous, cette crise révèle un déficit de confiance éthique dans le crédito, dont les ressorts sont liés à la structure institutionnelle des différents réseaux. II ne s'agit pas de réduire la question de la crise du trueque à une question de confiance : assurément, les origines possibles de la crise sont multiples. Ainsi, la littérature fait état de problèmes d'hygiène dans la préparation des aliments [Hintze et al., 2003, p. 65], de falsification et de vols de créditos émis par le RGT [ibid., p. 66] et d'une inflation relativement importante qui a touché l'ensemble des réseaux ${ }^{12}$. La massification de politiques d'assistance aux pauvres durant l'année 2002 a également pu contribuer à éloigner les populations les plus pauvres du trueque en leur offrant un revenu additionnel en peso. Il est enfin possible que cette crise ait été déclenchée (ou accentuée) par un retournement des alliances politiques nouées par les responsables locaux et nationaux des divers réseaux [Lacoste, 2003], car la massification du trueque a pu être vue comme l'avènement d'un pouvoir entrant en concurrence avec l'État dans le maintien de la paix sociale. Il est cependant difficile de comprendre quel fut le rôle exact de ces différents éléments, tant les éléments probants manquent. Aussi, I'argument développé dans cette section ne prétend pas proposer une interprétation définitive univoque de la crise du trueque : il s'agit plus modestement de souligner que celle-ci révèle l'existence d'un hiatus entre les projets politiques portés par les architectures institutionnelles des différents réseaux et les aspirations des participants ; ce faisant, le trueque souligne à quel point l'étude des crises monétaires ne peut faire l'impasse des dimensions politiques de la monnaie [Théret, 2007].

\subsection{Une nécessité méthodologique : dater la crise}

Deux documents permettent de dater la chute du nombre de participants entre mai et début septembre 2002. Le premier est la publication des actes de la Journée nationale du Trueque et de l'Économie solidaire, qui s'est tenue le 6 septembre 2002 : ce document mentionne à plusieurs reprises la « crise du trueque », tant dans les

\footnotetext{
${ }^{10}$ Ce terme désigne l'instauration en décembre 2001 de limites de retrait auprès des banques afin d'éviter une faillite du système bancaire, consécutive aux sorties massives de capitaux.

${ }^{11}$ Ces chiffres ont pour objet de donner un ordre de grandeur de la participation au trueque, mais ne sauraient être considérés comme des estimations fiables: Ovalles ne précise pas la méthodologie ni les hypothèses employées afin de construire son estimation.

${ }_{12}$ D'après nos enquêtes de terrain, en 2007 le rapport peso/crédito allait de 1/5000 à 1/10 000 dans l'unique feria subsistant à Buenos Aires, alors que celui-ci était fixé originellement à 1/1. En 2009, à Rosario ces mêmes rapports étaient compris entre 1/500 et 1/2500.
} 
chapitres émanant des chercheurs que dans ceux écrits par les représentants des principaux réseaux et dans les prises de parole des participants [Hintze, 2003]. La crise du trueque était donc déjà bien avancée au début du mois de septembre 2002. En outre, Ovalles [2002] nous informe que la forte décrue du nombre des participants du trueque n'a probablement pas commencé avant mai 2002. En effet, cette étude a été publiée en mai 2002 et ne mentionne à aucun moment une baisse du nombre de personnes, alors que son objet était précisément d'évaluer l'expansion quantitative du trueque. Or la chute de la participation au trueque semble avoir été si forte que cet événement ne l'aurait pas conduit à conclure en la croissance explosive du phénomène durant la première moitié de l'année 2002.

Il est possible de tester la robustesse de cette datation en la confrontant aux éléments permettant une appréhension indirecte de la crise. Ainsi, l'ensemble des éléments faisant directement référence à des problèmes locaux rencontrés par le trueque confirme que, dans les régions pour lesquelles ces informations sont disponibles, la situation s'est fortement dégradée entre juin et août 2002 [Hintze et al., 2003, p. 61-69; Lacoste, 2003, p. 9]. Les mois de juillet-août 2002 semblent avoir été les plus critiques, puisque les responsables du RGT annoncèrent puis mirent en œuvre le remplacement de 150 millions de créditos afin de juguler l'inflation qu'ils attribuaient à la falsification de leurs créditos. La Red Nacional de Trueque de Córdoba fit de même en août 2002. C'est également durant ces deux mois que Pablo Lacoste [2003, p. 26-28] situe le regain d'inflation du trueque dans la province de Mendoza.

Enfin, la datation proposée est cohérente avec l'attitude des gouvernements municipaux envers le trueque. Ceux-ci ont apporté un soutien croissant à l'expérience, à mesure que la participation à cette activité devenait de plus en plus massive [Luzzi, 2005, p. 131-157]. Ce soutien a atteint son apogée lorsque, durant la première moitié de l'année 2002, certaines municipalités ont accepté le règlement de tout ou partie des impôts municipaux en créditos (ceux-ci étant probablement utilisés par la suite afin de se procurer des aliments). Or aucun accord de ce type n'a été signé après juin 2002 [Hintze et al., 2003, p. 61-69]. II est probable que ce coup d'arrêt soit dû à la conjonction de la forte décrue du nombre de participants et de l'inflation qui toucha le trueque: dès lors, permettre le règlement des impôts municipaux en créditos n'était plus profitable, car il n'était plus possible d'accéder aux produits de base en grande quantité.

\subsection{Quelques facteurs à relativiser}

La crise qu'a connue le trueque en 2002 fut donc soudaine et n'a pas débuté avant mai 2002. Ces éléments permettent de relativiser le rôle de trois facteurs de crise parfois avancés dans la littérature : la réactivation de l'économie à partir de la fin de l'année 2002, les problèmes liés aux conditions d'hygiène de confection des aliments et la falsification de créditos. L'importance de la relance de l'économie doit d'abord être relativisée, car la crise du trueque intervient au plus profond de la crise économique : la tendance des principaux indicateurs macroéconomiques ne s'inversera qu'à partir de la fin de l'année 2002 [Heymann, 2006]. Ce facteur peut donc certes expliquer en partie la désaffection des secteurs populaires pour le trueque après 2002, mais pas la brutalité de la crise qu'il a connue au cours de l'année 2002. En outre, les problèmes 
d'hygiène et de falsification de créditos sont récurrents depuis janvier 2002, soit six mois avant le début probable de la crise du trueque [Hintze et al., 2003, p. 61-69].

La datation de la crise proposée ici permet également d'évaluer la pertinence de deux lectures de l'inflation du crédito qui n'intègrent pas la question de la confiance. La première appréhende ce phénomène comme l'importation dans la sphère du crédito de l'augmentation générale des prix libellés en pesos à travers deux processus simultanés : la hausse des prix libellés en peso se traduit d'une part par une « inflation par la demande », les denrées alimentaires les plus recherchées devant être préalablement acquises en peso ; d'autre part par une « inflation par les coûts », « relative à la répercussion de la hausse des coûts de production [en peso] sur le prix de vente [en crédito] » [Ould-Ahmed, 2008, p. 17]. Or le lien entre l'inflation qu'a connue le trueque et celle qui toucha le peso est loin d'être évident. En effet, on observe au cours de l'année 2002 une inflation annuelle du peso de 40,9\%, ce qui est très faible au regard de l'inflation qu'a connue le crédito. Mais surtout, l'inflation du peso a atteint son maximum au mois d'avril 2002 (10,4 \% mensuels), alors que la crise du trueque n'a pas débuté avant le mois suivant, au cours duquel l'inflation n'a été que de $4 \%$ (graphique 1).

Graphique 1 : Inflation mensuelle, peso, Argentine, 2002 (en \%)

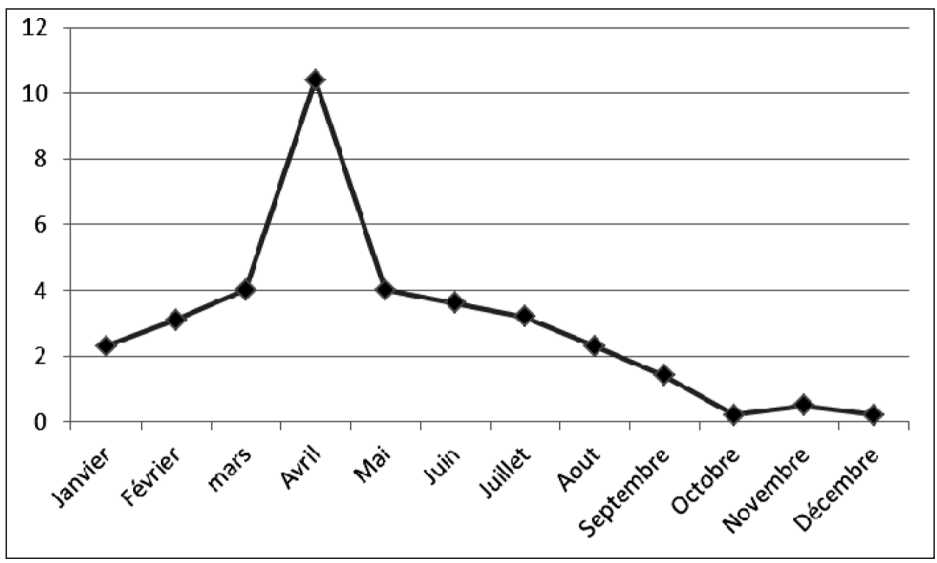

Source : élaboration propre à partir des données de I'INDEC - Indice des Prix à la Consommation.

Il convient également de ne pas interpréter l'inflation du crédito uniquement sur la base de l'évolution de la quantité de monnaie en circulation ramenée au volume de biens proposés. Selon les tenants de cette approche,

" c'est avant tout l'abondance de monnaie parallèle circulant qui était à l'origine de la hausse des prix. Dès qu'il devenait plus facile pour les participants d'obtenir des créditos - soit en les achetant "illégalement", soit en s'inscrivant à plusieurs nodos sans respecter les règles du système, la production pour le troc [trueque] décroissait et "les étals se vidaient". [...] En effet, l'équilibre entre le nombre de membres et la masse monétaire est rompu, le contrôle des prix disparaît et 
ceux-ci affichent une tendance à la hausse » (Luzzi [2005], p. 54 ; voir également Montillet [2006]).

La hausse de ce ratio (et, in fine, l'inflation) peut alors être expliquée par deux facteurs : d'une part, une progression du volume de produits proposés lors des ferias plus faible que celle du nombre de participants (les derniers arrivants étant supposés posséder un capital productif moindre); d'autre part, l'augmentation de la masse monétaire en circulation, engendrée par une surémission de créditos ou leur falsification. Selon cette interprétation, l'inflation que connut le trueque serait donc due à l'inadéquation technique des régimes de monnayage, qui n'auraient pas permis à la masse monétaire en circulation de s'adapter au volume des transactions.

Bien qu'ils ne le mentionnent pas explicitement, ces auteurs ont pour cadre de référence une lecture particulière de l'équation quantitative de la monnaie. Selon cette dernière, le produit de la masse monétaire $(\mathrm{M})$ et de sa vitesse de circulation $(\mathrm{V})$ est égal au niveau général des prix $(P)$ multiplié par la quantité de biens disponibles (Q) : $\mathrm{M} \times \mathrm{V}=\mathrm{P} \times \mathrm{Q}$. Dans cette optique, en effet, un accroissement de la quantité de monnaie en circulation (M) entraîne une augmentation des prix (P), tout comme la diminution des biens disponibles $(\mathrm{Q})$, toutes choses égales par ailleurs. Cependant, les analyses exposées au paragraphe précédent font une hypothèse implicite qu'il convient d'expliciter : elles estiment que la vitesse de circulation des moyens de paiement (V) est stable, puisque cette variable n'intervient pas dans leur raisonnement. Or il s'agit d'une variable cruciale, qui peut être analysée comme un indicateur du degré de confiance dont jouit le crédito : une vitesse de circulation qui augmente de manière vertigineuse est synonyme de perte de confiance dans le crédito puisque chacun souhaite se débarrasser au plus vite des moyens de paiement en sa possession [Orléan, 2007]. Ne pas prendre en considération les variations de la vitesse de circulation du crédito signifie donc écarter l'hypothèse d'une crise de confiance.

Or cette lecture particulière de l'équation quantitative de la monnaie permet difficilement d'expliquer la brutalité de l'effondrement du trueque : celle-ci serait due à une surémission de créditos soudaine d'une ampleur hautement improbable à partir de mai 2002, alors même que les fondateurs du RGT ont été accusés d'outrepasser les règles d'émission monétaire au moins dès 1999 et que l'affluence au trueque a continué à croître pendant plusieurs années. La soudaineté de la crise conduit donc à voir dans l'inflation de juillet-août 2002 la conjonction d'un probable accroissement de la masse monétaire en circulation et de l'accélération de la vitesse de circulation de la monnaie, qui pose, in fine, la question de la confiance. En l'absence de données quantitatives fiables concernant l'émission monétaire du RGT, il est difficile de préciser l'ampleur de l'accélération de la vitesse de circulation. Cependant, un examen attentif des événements de juillet-août 2002 révèle bel et bien un déficit de confiance éthique.

\subsection{Des déficits de confiance éthique ${ }^{13}$}

Cet argument prend appui sur un premier constat : la concomitance de l'octroi massif de subsides monétaires aux populations les plus pauvres (connus sous le nom de 
Planes Jefes y Jefas de Hogar) avec la crise du trueque. En effet, le nombre de bénéficiaires a littéralement explosé entre mai et juin 2002 : en deux mois, il est passé d'environ 5000 à 1500 000, avant d'atteindre et se stabiliser autour de 1750000 [Módolo, 2004, p. 8]. II semble donc que les planes aient fait partie des éléments déclencheurs de la crise du trueque, mais le lien de causalité liant ces événements demeure flou.

Il convient donc d'interroger ce que la prise de distance soudaine et massive des secteurs populaires vis-à-vis du trueque, à partir de la distribution des planes, enseigne quant à leur engagement au sein de celui-ci. Notre analyse prolonge celle de Leoni [2003, p. 84-85] en affirmant que cette prise de distance met au jour la précarité de l'engagement des plus pauvres au sein du trueque; elle l'enrichit en affirmant que cette précarité est reflétée par le défaut de fondement éthique de la confiance dans le crédito. Cette analyse prend appui sur les témoignages recueillis en 2007 dans le dernier nodo de Buenos Aires (affilié au RGT jusqu'en 2002), qui suggèrent qu'une crise de confiance éthique est venue parachever un défaut de confiance hiérarchique (surémission monétaire et falsifications de créditos) et méthodique (pénurie de certains biens). II est probable que la falsification des créditos ainsi que leur surémission au sein du RGT et la pénurie de certaines marchandises ont entraîné l'accroissement des prix de manière lente mais régulière, et ce, bien avant 2002. Devant la diminution du nombre de participants après l'implémentation des planes, les responsables de ce réseau ont décidé de remplacer les moyens de paiement en circulation par de nouveaux, plus difficiles à falsifier en juillet-août 2002 [Hintze et al., 2003, p. 67-68], imputant l'inflation à la falsification de créditos. Ils limitèrent cependant le montant ainsi remplacé à « une certaine quantité de créditos » [ibid., p. 67], considérant que le comportement des participants en possession d'une somme importante de créditos allait à l'encontre de la réciprocité (elle-même considérée comme opposée à "l'accumulation »). Ces participants sont d'ailleurs directement attaqués par Luis Nicolas Laporte, représentant du RGT lors de la Journée nationale sur le Trueque et I'Économie solidaire, lorsqu'il répondait aux critiques portant sur les modalités de remplacement des créditos du RGT :

"Certains participants ont accumulé des créditos, car dans le nodo qu'a visité hier Alberto Marino, "La Estación", il y a des producteurs de vêtements, comme cette productrice qui vendait [...] des vêtements au sein du club et qui a accumulé beaucoup de créditos. Elle avait 18000 créditos, ou 16000 créditos [...]. Enrique qui troquait des livres a fait de même. Des personnes comme cela il y en a beaucoup, beaucoup plus que ce que vous vous imaginez. » [Laporte, in Hintze (2003), p. 239.]

Cependant les personnes participant au trueque n'ont pas compris cette limitation. Le discours militant invoqué par Laporte leur était étranger et ils ont considéré qu'ils s'étaient fait spolier.

C'est ce que souligne Graciela, lorsqu'elle s'adresse à Luis Nicolas Laporte en réaction à la citation précédente :

"Je voudrais simplement lui dire que je crois que ce n'est pas nous qui nous trompons, car j'ai également accumulé, comme tant de gens, des créditos et je ne l'ai pas fait en spéculant, je l'ai fait en travaillant [...]. Je crois donc que la plus 
grande erreur dans laquelle le RGT tombe [...] est de penser que nous avons mis en œuvre une politique de spéculation et que nous réunissions et accumulions des créditos pour on ne sait quelle raison. Les créditos que nous avons générés, nous les avons générés en travaillant, donc [les modalités d'échange des nouveaux billets que vous proposez], c'est une escroquerie de plus, exactement comme ce qui se fait dans l'économie formelle [corralito], voire pire. » [Graciela Parvis, in Hintze (2003), p. 240.]

Dans la littérature, beaucoup de participants évoquent cet épisode pour expliquer leur éloignement du trueque ou, du moins, leur moindre investissement dans cette activité [Leoni, 2003, p. 72]. II est possible de qualifier cet épisode de crise de confiance éthique, car le fondement éthique mis en avant par les responsables de ce réseau afin de justifier la règle de remplacement des anciens créditos ne correspondait pas à la représentation que se faisaient les participants de leur engagement au sein du RGT. Autrement dit, les modalités d'émission et de distribution des nouveaux moyens de paiement étaient le reflet d'un projet politique loin d'être partagé par tous ; elles furent en conséquence considérées comme illégitimes.

L'analyse qui vient d'être proposée ne peut pas être transposée terme à terme au RTS : un remplacement des moyens de paiement d'une ampleur analogue est peu probable, dans la mesure où l'émission monétaire y était décentralisée. La crise du RTS est souvent lue comme étant une conséquence de l'inflation qui toucha le RGT : son origine serait étrangère au RTS et aurait en quelque sorte été importée en son sein par le RGT [Primavera, 2003 ; Cortesi, 2003 ; Pérez-Lora, 2003]. II convient donc de porter une attention particulière à la transmission de l'inflation du RGT vers le RTS, telle qu'elle apparaît dans le témoignage de Marita :

Marita : Les créditos [du RGT] apparaissaient [dans les ferias du RTS], mais ce fut en cachette. [...] Si l'on voulait acheter un article qui coûtait cher, au lieu de produire pour se procurer les créditos [RTS], on pouvait aller acheter les créditos du RGT et les gens les acceptaient comme s'il s'agissait de la même chose [que ceux du RTS] ; ils ne comprenaient pas la question [...] de l'inflation [...].

Moi : Pardon, comment le RTS a-t-il réagi lorsque vous vous êtes rendu compte que les créditos [RGT et RTS] étaient mélangés [lors des ferias]?

Marita : Lorsque nous nous en sommes rendu compte, nous faisions tout un travail de prise de conscience durant la feria, mais cela ne fonctionna pas [no habia manera] ; ce fut une époque terrible. [Entretien avec Marita, novembre 2009; voir également Pérez-Lora, 2003, p. 226-227.]

Cet extrait d'entretien suggère que le « mélange de créditos » est à l'origine de la propagation de la crise du RGT vers le RTS et que cette pratique met au jour le manque de confiance éthique dont ont pu souffrir les créditos émis par le RTS. En effet, Marita reconnaît qu'elle n'a pas été en mesure de faire comprendre aux participants des ferias de Capitán Bermúdez l'importance de ne pas « mélanger les créditos » émis par les différents réseaux. Or en acceptant dans les ferias du RTS un crédito dont l'émission était centralisée, cette pratique remettait en cause la caractéristique fondatrice du 
RTS : la décentralisation de l'émission monétaire. La raison d'être du RGT était donc loin d'être partagée par l'ensemble des participants et ces derniers la mirent à mal. Dès lors, il était impossible de faire appel à la décentralisation de l'émission monétaire en tant que principe supérieur, faisant autorité et par là à même d'orienter les comportements individuels, afin de bannir l'usage des créditos émis par le RGT au sein du RTS.

\section{Conclusion : pour une étude des fédéralismes monétaires}

La lecture de l'histoire du trueque proposée ici permet de tirer deux enseignements de portée générale. L'expérience du RGT puis du RTS souligne d'abord que le fédéralisme monétaire suppose des négociations constantes afin de satisfaire ses deux contraintes constitutives. La première est celle de la pluralité des émetteurs des moyens de paiement : la décentralisation de l'émission monétaire est le corollaire de la reconnaissance de l'hétérogénéité des besoins monétaires sur le territoire. La deuxième est l'unicité du système de compte, afin d'assurer sur l'ensemble du réseau la validité au pair des moyens de paiement émis localement. Or ces deux contraintes ne sont pas nécessairement remplies simultanément : d'une part, la pluralité des émetteurs de moyens de paiement peut se traduire par l'apparition de taux de change internes non paritaires ; d'autre part, l'unique architecture institutionnelle permettant de garantir l'unicité du système de compte en toute circonstance est la centralisation de l'émission monétaire, qui est contraire au principe de fédéralisme monétaire. Dans le cas du RGT puis du RTS, cette tension a été résolue à travers la soumission des zones (entités fédérées) à un ensemble de règles communes encadrant l'émission monétaire, cristallisées dans la constitution du balance. II n'en demeure pas moins que la tension entre unicité du compte et pluralité des émetteurs de moyens de paiement est inhérente au fédéralisme monétaire. Ce constat permet de jeter un regard neuf sur le RGT (après 2001) et le RTZO : la centralisation extrême de l'émission monétaire les conduit à ne satisfaire qu'une seule des contraintes constitutives du fédéralisme monétaire, sacrifiant la pluralité des émetteurs afin d'assurer l'unicité du compte. II s'agit là d'une forme extrême de centralisation que ne connaissent pas la plupart des systèmes monétaires contemporains.

L'histoire du trueque incite également à adopter un regard critique vis-à-vis des approches instrumentales de la monnaie, qui voient dans celle-ci un instrument unifié visant à faciliter les échanges [Orléan, 2007 ; Blanc, 2000]. En effet, elles ne permettent de rendre compte d'aucune des contraintes constitutives du fédéralisme monétaire. Ainsi, à notre connaissance, elles n'ont développé aucune réflexion relative à la décentralisation de l'émission monétaire. Mais surtout, en adhérant à des théories de la valeur qui n'offrent aucun rôle à la monnaie dans la fixation des prix relatifs, ces approches ne permettent pas de traiter la question de l'unicité du compte. L'importance de cette question dans le RGT puis le RTS souligne au contraire que la monnaie est d'abord un système d'évaluation des dettes et qu'aucune transaction n'est possible sans norme commune d'évaluation. Ainsi, la lecture de l'histoire du trueque proposée ici prend le contre-pied des approches instrumentales en soulignant la dimension politique de la monnaie, puisque la diversité des modalités 
d'émission des moyens de paiement est l'expression monétaire de projets de société concurrents.

La réflexion proposée ici est cependant embryonnaire, car elle est basée uniquement sur l'histoire du trueque. Ainsi, le RGT et le RTS doivent être considérés comme les représentants de l'une des formes possibles d'un principe monétaire plus vaste d'unité (du système de compte) dans la diversité (des émetteurs de moyens de paiement). Afin d'enrichir cette approche, nous suggérons de multiplier les travaux empiriques visant à prendre connaissance des multiples déclinaisons possibles du principe de fédéralisme monétaire, puis de les mobiliser conjointement afin d'étayer I'hypothèse de contrainte en vue de la cohérence institutionnelle entre fédéralismes politique et monétaire, suggérée par Julio Olivera [1992, p. 17]. Dans cette perspective, une première étape consisterait à analyser conjointement l'histoire du trueque, des monnaies fiscales provinciales et de la politique monétaire de la Banque centrale de la République argentine à travers le prisme de la question du fédéralisme, sur la base de la littérature existante [Théret et Zanabria, 2007 ; Théret, 2012].

\section{Bibliographie}

AgliettA M. et al. (1998), «Introduction », in M. Aglietta et A. Orléan (dir.) La monnaie souveraine, Odile Jacob, Paris, p. 9-31.

Arnsperger C. (2011), " Monetary federalism and the recovery of Swiss sovereignty ", ECSA-Switzerland Conference Aspekte der Souveränität in den Beziehungen der Schweiz zur Europäischen Union, Bâle, 11 décembre.

BLAnC J. (2000), Les monnaies parallèles, L'Harmattan, Paris.

CORTESI J. (2003), « Red de Trueque Solidario (RTS) », in S. Hintze (dir.) Trueque y economia solidaria, Prometeo Libros, Los Polvorines, p. 181-196.

EchavarRI, L. (2003), « Contexto de necesidad y aspiraciones de transformación en el ámbito asociativo del Trueque ", Concours CLACSO: Movimientos sociales y nuevos conflictos en América Latina y el Caribe, Buenos-Aires.

GómEZ G. (2008), Making markets. The institutional rise and decline of the Argentine Red de Trueque, Thèse de doctorat en études du développement, Institute of Social Studies, La Haye.

González Bombal I. (2002), « Sociabilidad en clases medias en descenso: experiencias en el trueque ", in L. Beccaria et al. (dir.) Sociedad y sociabilidad en la Argentina de los 90, Biblios, Buenos-Aires, p. 97-136.

Heymann D. (2006), « Buscando la tendencia: crisis macroeconómica y recuperación en la Argentina ", Working paper CEPAL - SERIE estudios y perspectivas, $\mathrm{n}^{\circ} 31$.

Hintze S. (dir.) (2003), Trueque y economia solidaria, Prometeo Libros, Los Polvorines.

Hintze S., Federico Sabaté A. et Coraggio J.-L. (2003), « Documento base de la Jornada Nacional sobre Trueque y Economía Solidaria », in S. Hintze (dir.) Trueque y economia solidaria, Prometeo Libros, Los Polvorines, p. 19-86

KESSLER G. (1999) «L'expérience de paupérisation de la classe moyenne argentine », Cultures et Conflits, $n^{\circ} 35$, p. 71-93.

Lacoste P. (2003), « La crisis socioeconomica argentina y las respuestas sociales : las redes de clubes de trueque », Revista Confluencia, n 1, p. 115-158. 
Laporte L.-N. (2003), « La Red Global del Trueque (RGT) », in S. Hintze (dir.) Trueque y economia solidaria, Prometeo Libros, Los Polvorines, p. 163-179.

LEONI F. (2003), Ilusión para muchos, alternativa para pocos. La práctica del trueque en los sectores populares, Mémoire de diplôme en politique sociale, Universidad nacional de General Sarmiento.

Luzzı M. (2005), Réinventer le marché ? Les clubs de troc face à la crise en Argentine, L'Harmattan, Paris.

McClanahan R. (2003), Making Money From the Bottom-Up: The Clubes del Trueque and Local Development in Argentina. Mémoire de Master en sciences politiques, University of Texas at Austin.

Módolo C. (2004), "Los peligros institucionales del plan Jefes y Jefas de Hogar ", Investigaciones en la Facultad de Ciencias Económicas y Estadística, Universidad de Buenos Aires, novembre.

Montillet L. (2006), «Et pour quelques créditos de plus... », in J. Blanc (dir.) Exclusion et liens financiers. Monnaies sociales. Rapport 2005-2006, Economica, Paris, p. 401-422.

OLIVERA J. (1992), "Banca central, federalismo económico y constitución monetaria », Nuevas Propuestas, revista de la Universidad Católica de Santiago del Estero (juin), p. 7-17.

OrLÉAN A. (2007), «Crise de la souveraineté et crise de la monnaie : I'hyperinflation allemande des années 1920 », in B. Théret (dir.) La monnaie dévoilée par ses crises, Éditions de l'EHESS, Paris, p. 187-229.

Ould-Ahmed P. (2008), « Les formes du politique dans les "clubs de troc" en Argentine ». Working Paper, 2008-1 série M-F, Association Recherche et Régulation.

Ovalles E. (2002), « Argentina es el pais del mundo en el cual el fenomeno del trueque tiene mayor dimension social ", Centro de Estudios Nueva Mayoria, Buenos Aires.

Paraje G. (2005), « Crisis, reforma estructural y... nuevamente crisis : desigualidad y bienestar en el Gran Buenos Aires ». Desarrollo economico, vol. 45, n 179, p. 373-402.

ParRY J. P. et Bloch M. (dir.) (1989), Money and the morality of exchange, Cambridge University Press, Cambridge.

Pérez LoRa C. (2003), « Región Mar y Sierras - RTS», in S. Hintze (dir.) Trueque yeconomia solidaria, Prometeo Libros, Los Polvorines, p. 207-228.

Powel. J. (2006), « Capitalisme insignifiant, capitalisme en perfectionnement ou post-capitalisme ? Enseignements des expériences argentines de troc », in J. Blanc (dir.) Exclusion et liens financiers. Monnaies sociales. Rapport 2005-2006, Economica, Paris, p. 423-442.

Primavera H. (2003), «Riqueza, dinero y poder: el efímero "milagro argentino" de las redes de trueque », in S. Hintze (dir.) Trueque y economia solidaria, Prometeo Libros, Los Polvorines, p. 121-144.

Primavera H., Covas H. et de Sanzo C. (1998), Reinventando el mercado: la experiencia de la Red Global de Trueque en Argentina, Bernal, PAR. 
RAVERA R. (2003), «Exposición de Rubén Ravera sobre aspectos relativos a la moneda de las redes de trueque », in S. Hintze (dir.) Trueque y economia solidaria, Prometeo Libros, Los Polvorines, p. 145-159.

Salles P.-O. (2006), "Entre survie et engagement politique, les avatars du projet militant du trueque argentin », in J. Blanc (dir.) Exclusion et liens financiers. Monnaies sociales. Rapport 2005-2006, Economica, Paris, p. 481-495

Sampayo F. (2003), «Club del Trueque Zona Oeste», in S. Hintze (dir.) Trueque yeconomia solidaria, Prometeo Libros, Los Polvorines, p. 197-206.

SERVET J.-M. (dir.) (1999), Une économie sans argent : les systèmes d'échange local, Le Seuil, Paris.

SERVET J.-M. (2009), Le Kaléidoscope des sciences sociales : introduction à l'interdisciplinarité, Genève, IHEID, miméo.

Servet J.-M., Théret B. et YILDIRIM Z. (2008), « Universalité du fait monétaire et pluralité des monnaies : de la confrontation coloniale à la rencontre des sciences sociales», dans E. Baumann et al. (dir.) Argent des anthropologues, monnaie des économistes, L'Harmattan, Paris, p. 167-207.

THÉRET B. (dir.) (2007), La monnaie dévoilée par ses crises, Éditions de l'EHESS, Paris.

Théret B. (2008), « Les trois états de la monnaie. Approche interdisciplinaire du fait monétaire », Revue économique, vol. 59, n 4, p. 813-841.

THÉRET B. (2012), " The BOCADE, currency of the Argentinian province of Tucuman between 1985 and 2003: a case of monetary complementarity caused by locality ", International workshop De-teleologising history of money and its theory, Institute of Advanced Studies on Asia, University of Tokyo, February, 16-17.

Théret B. et Zanabria M. (2007), « Sur la pluralité des monnaies publiques dans les fédérations », Économie et institutions, nos 10-11, p. 9-66.

Veron Ponce M. B., Heredia L. et Sanchez J. (2003), « Responde el Trueque en la Red de Prosumidores Catamarca al modelo de la socioeconomia solidaria? », El Trueque, expresión de Economía Solidaria, San Fernando del Valle de Catamarca, juin.

SaIAG H. (2011), Le trueque argentin au prisme de la dette : une socioéconomie des pratiques monétaires et financières, Thèse de doctorat en science économique, Université Paris-Dauphine.

Zelzer V. (2005 [1994]), La signification sociale de l'argent, Le Seuil, Paris. 